\title{
On Stability of Finite Control Set MPC strategy for Multicell Converters
}

\author{
Ricardo P. Aguilera and Daniel E. Quevedo \\ School of Electrical Engineering and Computer Science \\ The University of Newcastle, Australia \\ Email:aguilera.ricardo@gmail.com,dquevedo@ieee.org
}

\begin{abstract}
Finite Control Set Model Predictive Control has emerged as a promising control technique for Power Converters due to the fact that the inherent non-linearity of these systems can be taken into account. In addition, this strategy directly provides the switching action to be applied to the converter; consequently, additional modulation stages are not required. Despite these advantages, stability for this class of predictive control technique remains as an open problem. In the present work, we provide insights into the closed-loop stability for the Finite Control Set Model Predictive Control. As an illustrative example, we apply these stability guidelines to a Multicell Converter.
\end{abstract}

\section{INTRODUCTION}

Utilization of power electronics converters has considerably increased recent years covering a wide range of industrial applications at different rated powers (low, medium and high voltage). For this reason, power converters have been in constant development in order to not only obtain new advantageous architectures but also to improve control techniques to achieve superior performance. With respect to the last point, predictive control strategies have emerged as a promising control technique. These methods hold many advantages compared to traditional PWM control schemes [1, 2].

Different predictive control approaches have been used to handle power converters. One of the first to be applied was the, so-called, dead-beat control method [3]. This strategy basically assumes that the converter can generate a continuous output voltage. Thereby, by using a predictive model of the system, one can determine the required value of output voltage in order to track the desired current reference. Subsequently, this voltage is converted into a form that can be implemented in practice using a modulation stage. A disadvantage of this technique is that system constraints are difficult to incorporate.

Another important approach is via Model Predictive Control (MPC) or receding horizon control (RHC) [4]. This technique calculates the control action by solving, at each sampling instant, an optimal control problem which forecasts, over a finite horizon, the future system behavior. To achieve this, the current system state is taking into account. This generates an optimal control sequence. Finally, the control action to be applied to the plant is the first element of this sequence. The main advantage of MPC is that constraints and non-linearities can be included. Nevertheless, a large amount of calculation is required to obtain the optimal control sequence. For this reason, MPC is not widely used in practical power converters.
To address this problem a Generalized Predictive Control (GPC) method can be used [5]. Here, a linear controller is obtained by analytically solving the optimization problem. However, solving the optimal problem when constraints are included is a formidable task. Both MPC and GPC provide a no-quantized voltage as a solution, so in practice, a modulation stage is still required.

One of the most attractive predictive algorithm for power converters is the so-called, Finite Control Set Model Predictive Control (FCS-MPC). The main advantage of this predictive strategy comes from the fact that switching actions are expressly tacking into account. Consequently, modulation stages (to handle the switching elements) are not required. This strategy directly considers the switching elements as the control input. Using a discrete model for the system to forecast the future behavior of the system state, a cost function is evaluated at each sampling period by exploring the allowable switch combinations. Finally, the switching action to be applied in the next sampling instant is the one that minimizes the proposed cost function.

Despite the good performance that FCS-MPC in principle offers, there remain several open problems. One important issue is to define a proper cost function. In $[1,2]$ two kinds of cost functions have been proposed for the FCS-MPC strategy. The first one considers the sum of the absolute value for each individual system error. A second cost function uses the Euclidean norm of the system error. Nevertheless, any criterion has been given to define it. Another issue is that closed-loop stability for FCS-MPC scheme has not been established. The usual approach to guarantee stability in MPC is to consider that the value function of the optimal problem is a candidate Lyapunov function $[4,6]$. For switched systems, an extension of this tool to this, so-called, Multiple Lyapunov Function (MLF) has been explored [7]. This technique evaluates the average value of multiple Lyapunov functions during the period that each subsystem is applied in order to determine the optimal switching trajectory.

In the present work we provide further insight into the conditions necessary to achieve closed-loop stability for Finite Control Set Model Predictive Control algorithm when it is applied using constant references, i.e., DC-DC converters. As an illustrative example, the proposed stability guidelines will be used to control a Multicell Converter. This kind of topology presents the problem that one need to control not only the 
output voltage but also the internal capacitor voltages. This have been easily solve by using FCS-MPC algorithm [8].

\section{PRoblem DESCRIPTION}

In this section we present preliminary ideas related to power converter models. In addition, we describe the FCS-MPC strategy in more detail as a base for a future stability analysis.

\section{A. Switched Systems}

In general, power converters can be classified as Switched Systems due to the fact that a change in the switching elements implies a change between two different subsystems. These subsystems can be either linear or non-linear. A switched system can be mathematically defined by

$$
\dot{x}(t)=f(x(t), u(t))
$$

where $x(t) \in \mathbb{X} \subseteq \mathbb{R}^{n}$ is the system state and $u(t) \in \mathbb{R}^{m}$ is the controlled input which, in this case, represents the switching elements. Each element of the input can take only two values, $u_{j}(t) \in\{0,1\}$. Consequently, in this kind of system, each element from the input is restricted to belong to a finite set of $q=2^{m}$ elements, namely $u(t) \in \mathbb{U}=\left\{u_{1}, \cdots, u_{q}\right\}$. Once a control input $u_{j}$ is applied, one can represent the system (1) in terms of its associated subsystem as:

$$
\dot{x}(t)=f_{j}(x(t)), \quad j \in\{1, \cdots, q\} .
$$

It is important to remark that the input remains constant during a sample period $h$ and can only change at each sampling instant $k$. Therefore, the system (1) can be represented in discrete time via a model of the form:

$$
x[k+1]=g(x[k], u[k]) .
$$

When a pre-determined control input $u_{j}$ is applied, the system can be represented by its associated linear system as

$$
x[k+1]=g_{j}(x[k]), \quad j \in\{1, \cdots, q\} .
$$

Using this system definition, the design problem can be stated as finding an algorithm which provides an appropriated control input sequence

$$
\vec{u}=\{u[k], \cdots, u[k+N]\}
$$

which allows one to reach the desired reference, $x^{\star}(t)$. Fig. 1 depicts this formulation of this problems.

\section{B. FCS-MPC Strategy}

As mentioned above, the FCS-MPC strategy is implemented in discrete time with sampling frequency $f_{s}=h^{-1}$. At each discrete instant $k$, a measurement of the system state $x[k]$ is taken and then a cost function (which considers the system error) is evaluated for each control input element $u_{j}$.

In this work we will focus on a cost function using a Euclidean norm because it is mathematically more tractable. Therefore, the proposed cost function is expressed via:

$$
J[k](\vec{u}[k], x[k])=\sum_{l=k+1}^{k+N}\left\|x[l]-x^{\star}[l]\right\|_{P}^{2},
$$

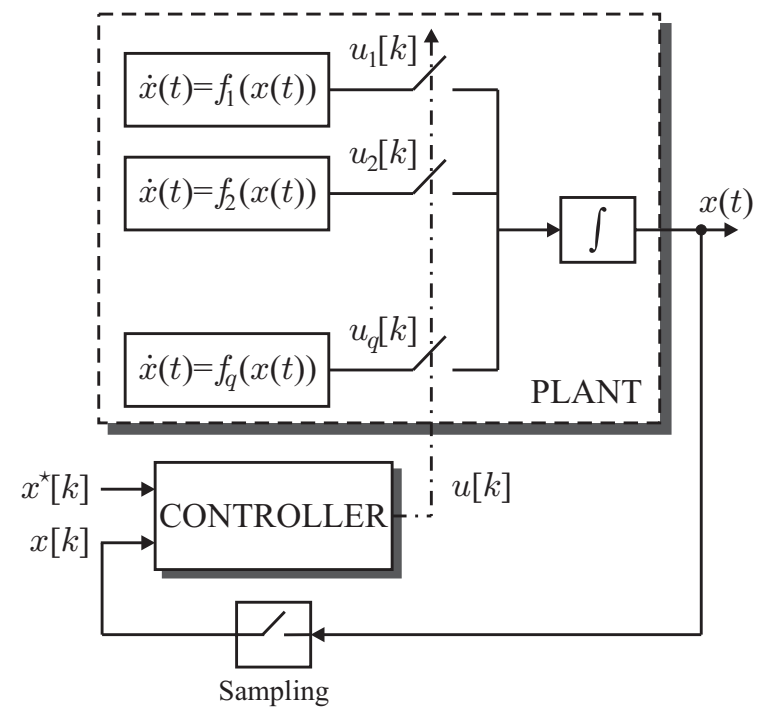

Fig. 1. Switched System diagram

where $\|\cdot\|_{P}$ stands for

$$
\left\|x[l]-x^{\star}[l]\right\|_{P}^{2} \triangleq\left(x[l]-x^{\star}[l]\right)^{T} P\left(x[l]-x^{\star}[l]\right),
$$

and

$$
P=\operatorname{diag}\left\{\lambda_{i}, \ldots, \lambda_{n}\right\} .
$$

Here, $\lambda_{i}$ are nonnegative scalar tuning parameters. The decision variables are:

$$
\vec{u}[k] \triangleq\{u[k+1], \cdots, u[k+N]\}
$$

The vector $\vec{u}[k]$, thus, contains tentative switching actions over a horizon of finite length $N$. The optimal switching action to be applied at time $k+1$, namely $u_{\text {opt }}[k]$ will be the first element of $\vec{u}[k]$. Then, at the next instant, $k+1$, the cost function $J[k+1](\vec{u}[k+1], x[k+1])$ is minimized using fresh state measurements. This gives $u_{o p t}[k+1]$ and so on.

\section{Stabilization CONDitions FOR FCS-MPC}

In this section we investigate the stabilization of power converters when the FCS-MPC strategy is used. We will split the problem in two parts. Firstly, we will give the conditions that the system must satisfy in order to "point towards" the reference. Secondly we will define a region of admissible error in which the system will entre and remain. Such region is called positive invariant set [9].

\section{A. Cost Function Definition}

We first define a continuous time candidate Lyapunov function from (6) considering a prediction horizon of $N=1$ and a constant reference $x^{\star}$ via:

$$
V(t)(x(t))=\left(x(t)-x^{\star}\right)^{T} P\left(x(t)-x^{\star}\right) .
$$

From (8) one can say that $P=P^{T}>0$, so it is clear that

$$
V(t)(x(t))>0 \forall x \neq x^{*} .
$$


It is important to notice that

$$
J[k](x[k])=V(h k)(x(h k)),
$$

where $t=h k$ and $h$ stands for the sample period.

To guarantee that the system is asymptotically stable, we require $V(h(k+1))-V(h k)<0$, the following condition must be satisfied for small time increments:

$$
\frac{d V(t)}{d t}=2\left(x(t)-x^{\star}\right)^{T} P f_{j}(t)<0 .
$$

Due to the fact that this condition depends on the dynamics of the associated subsystem, $f_{j}$, we need to ensure that the optimal switching action $u_{j}(k)$ not only minimize the cost function $J[k]$ but also satisfies the condition (13).

\section{B. Invariant Set Design}

Decreasing monotonically the value of the cost function $J(t)$ is only possible when the system is far from the reference $x^{\star}$. If, at a sampling instant $k$ a control input $u_{j}(k)$ is applied producing a vector field $f_{j}$ which satisfies (13), and also within the sample period between $k$ and $k+1$ some of the system variables cross the reference, it can happen that the condition (13) will be not satisfied at $k+1$. This situation is depicted at Fig. 2.

To deal with this issue, we define a region $\mathcal{D}$ around the reference $x^{\star}$ in which the cost function $J(t)$ cannot be decreased. [9]).

Formally, a positive invariance set is defined as follows (see

Definiton 1: (Positive Invariant Set) A set $\mathcal{D} \subseteq \mathbb{X}$ is said to be positively invariant with respect to (1) if, among all the possible solutions originated form $x(0) \in \mathcal{D}$, there exist at least one which remains inside $\mathcal{D}$, it means $x(t) \in \mathcal{D}$ for $t>0$.

To explain the idea of invariant set, we note that the cost function $J[k]$ represents an ellipsoid in the Euclidean space having the reference $x^{\star}$ as its center. The factors $\lambda_{i}$ give the eccentricity of this ellipsoid. When the cost function is decreased, a sequence of ellipsoids smaller than the previous one will be generated.

Let $\mathcal{D}$ the smallest ellipsoid in which the system will be confined. This region can be represented via:

$$
\mathcal{D}=\left\{x \in \mathbb{X}:\left\|x(t)-x^{\star}\right\|_{P}^{2} \leq \varepsilon\right\} .
$$

If the control input sequence satisfies condition (13), the state system always will "point towards" the reference. Therefore, the maximum deviation that each system variable, $x_{i}$, can have will be when the system exactly meets the reference $x^{\star}$. Thus, this maximum deviation vector $\delta_{i}$ can be obtained by:

$$
\delta_{i}=\max _{j}\left\{g_{i j}\left(x_{i}^{\star}\right)\right\}
$$

where each element of $g_{i j}\left(x_{i}^{\star}\right)$ is obtained from the discrete model (3). Now, the smallest ellipsoid will be that one which

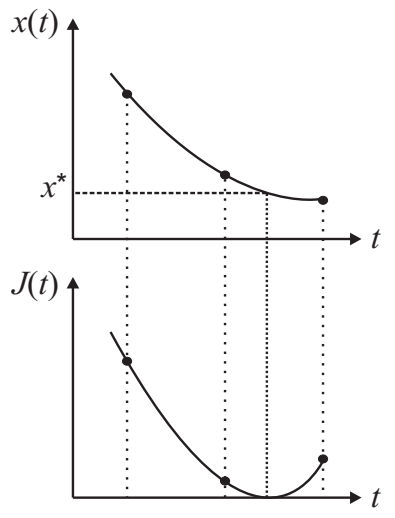

Fig. 2. System state error produced by FCS-MPC near to the reference $x^{\star}$.

contains all the maximum deviations. Therefore, the "radius" of this ellipsoid can be expressed via:

$$
\varepsilon=\max _{i}\left\{\lambda_{i} \delta_{i}^{2}\right\}
$$

It is important to notice that this ellipsoid depends on the parameters of the system and the sample period $h$. Assuming that the system parameters cannot be modified, $\varepsilon$ will define the maximum system error. Otherwise, if one can impose an admissible error for the system, one must find the maximum sample period $h$ by (15) and (16) to achieve this requirement.

\section{ILLUSTRATIVE EXAMPLE}

As a motivating example, we consider a Multicell converter working as a DC-DC converter. This class of converter utilizes electrically floating capacitors to synthesize the intermediate voltage levels required [10]. It is for this reason that this topology is also known as a Flying Capacitor Converter (FCC). Recently, predictive control formulation have been applied to FCC achieving good performance [8, 11-13].

Fig. 3 shows a schematic of an $n$-cell FCC. One can see that each cell $i$ consists of a capacitor $C_{i}$ and two switching elements $S_{i}$ and $\bar{S}_{i}$ which, at the same instant act in a complementary fashion to avoid a short circuits. The converter is connected to a mains dc-voltage source $V_{d c}$ and must provide the desired electrical current $i_{L}$ to the inductive load by commuting the switch states. On the other hand, the internal capacitor voltages, $v_{c i}$, must be controlled to achieve the, so-called, balanced capacitor voltages condition in order to spread the blocking voltage equitably among the switching elements.

\section{A. System Model}

Since the two switches in the same cell work in complementary fashion, the state of this pair of switches can be represented only by the state of one of them. This state can 
be expressed as follow:

$$
S_{i}(t)= \begin{cases}0 & \text { if } S_{i}=0 \text { and } \bar{S}_{i}=1, \\ 1 & \text { if } S_{i}=1 \text { and } \bar{S}_{i}=0 .\end{cases}
$$

for all $i \in\{1, \ldots, n\}$.

Taking into account that the capacitor voltages in the $n_{t h}$ cell is imposed by the main dc-voltage, we will need to control the $n-1$ remainder capacitor voltages plus the output current.

From this, for a $n$-cell FCC, we will consider the state variables as: $x_{i}=v_{c i}(t)$ for $i \in\{1, \ldots, n-1\}$ and $x_{n}=i_{L}(t)$. Moreover, the control input will be: $u_{i}=S_{i}(t)$ where $u_{i}(t)$ $\in\{0,1\}$. Finally, a simple dynamic model of the system can be represented via:

$$
\begin{aligned}
& \dot{x}_{i}=-\frac{1}{C_{i}} x_{n}\left(u_{i}-u_{i+1}\right) \quad i \in\{1, \ldots, n-1\}, \\
& \dot{x}_{n}=-\frac{R}{L} \cdot x_{n}+\frac{1}{L} v_{a o} .
\end{aligned}
$$

where the converter output voltage is defined by:

$$
v_{a o}=V_{d c} \cdot u_{n}+\sum_{i=1}^{n-1} x_{i}\left(u_{i}-u_{i+1}\right)
$$

Due to the fact that the FCS-MPC algorithm operates in discrete time with a fixed sampled period $h$, it is necessary to obtain a discrete time model of the system. To do this, we use a forward Euler approximation to the capacitor voltage equation. The output current equation is transformed into discrete time using a zero order hold approximation.

Finally, the discrete model for an $n$-cell FCC can be expressed via:

$$
\begin{aligned}
x_{i}[k+1] & =x_{i}[k]-\frac{h}{C_{i}} x_{n}[k]\left(u_{i}[k]-u_{i+1}[k]\right) \\
x_{n}[k+1] & =K_{a} x_{n}[k]+K_{b}\left(v_{a o}[k]\right) .
\end{aligned}
$$

where:

and

$$
\begin{aligned}
K_{a} & =e^{-R h / L}, \\
K_{b} & =\left(1-K_{a}\right) / R,
\end{aligned}
$$

$$
v_{a o}[k]=V_{d c} \cdot u_{n}[k]+\sum_{i=1}^{n-1} x_{i}[k]\left(u_{i}[k]-u_{i+1}[k]\right) .
$$

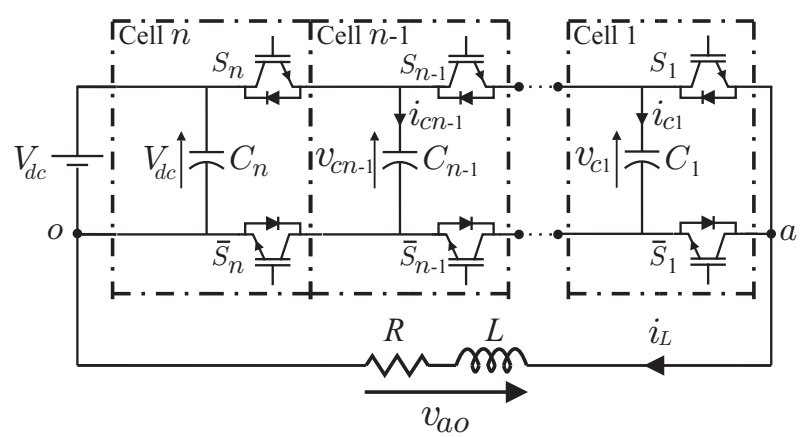

Fig. 3. $n$-cell Flying Capacitor Converter

\section{B. Stability Analysis for a 2-cell FCC}

For clarity of presentation, we will analyze the FCS-MPC strategy applied to a 2-cell FCC. In this case it is necessary to control the output current $i_{L}(t)$ and one capacitor voltage $v_{c}(t)$. We utilize two complementary switches. These generate $q=4$ different subsystems

$$
u(t) \in\{\{0,0\},\{0,1\},\{1,0\},\{1,1\}\} .
$$

If we consider the ellipsoidal cost function, see Fig. 4, we can represent the system state by:

$$
\begin{aligned}
& x_{1}(t)-x_{1}^{\star}=\delta_{1} \cos (\theta) \\
& x_{2}(t)-x_{2}^{\star}=\delta_{2} \sin (\theta)
\end{aligned}
$$

Then, the cost function can be represented via:

$$
J[k]=\lambda_{1} \delta_{1}^{2} \cos ^{2}(\theta)+\lambda_{2} \delta_{2}^{2} \sin ^{2}(\theta) .
$$

To obtain a constat value of $J[k]=\delta^{2}$ along the trajectory of $\theta$, we need to redefine the system state as:

$$
\begin{aligned}
& x_{1}(t)-x_{1}^{\star}=\frac{\delta}{\lambda_{1}} \cos (\theta) \\
& x_{2}(t)-x_{2}^{\star}=\frac{\delta}{\lambda_{2}} \sin (\theta)
\end{aligned}
$$

Later, we will determine conditions for what the system can satisfy the condition presented in (13).

Firstly, for the input control $u_{1}$ we have:

$$
-\left(x_{2}(t)-x_{2}^{\star}\right) x_{2}(t)<0 .
$$

Independent of the number of cells, capacitor voltages are restricted to belong to $v_{c} \in\left(0, V_{d c}\right)$; therefore, the output current can take values only in this range: $i_{L} \in\left(0, \frac{V_{d c}}{R}\right)$. Hence, $x_{2}(t)>x_{2}^{\star}$ which implies that $\frac{\delta}{\lambda_{2}} \sin (\theta)>0$. So, one can say that the switch combination $u_{1}$ can be applied in an angular range of $\theta \in(0, \pi)$.

Next, if we consider that the control input $u_{4}$ is applied, then

$$
-\left(x_{2}(t)-x_{2}^{\star}\right)\left(x_{2}(t)-\frac{V_{d c}}{R}\right)<0 .
$$

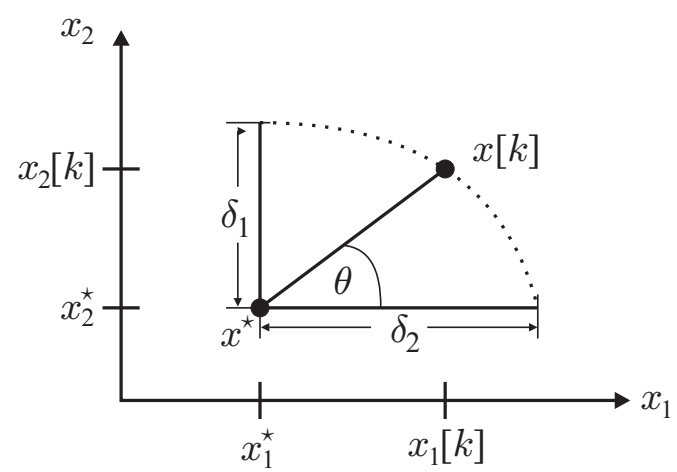

Fig. 4. Ellipsoidal representation of the system 
Here, constraint (14) is satisfied whenever $\theta \in(\pi, 2 \pi)$. A similar analysis can be carried out for the remaining switching states (details are omitted for the sake of brevity).

Finally, given an initial condition for the system in which $x_{1} \in\left(0, V_{d c}\right)$ and $x_{2} \in\left(0, \frac{V_{d c}}{R}\right)$ one can guarantee that the system can be stabilized by using FCS-MPC algorithm.

\section{Simulation Results}

To verify the performance of the methodology describe above, simulation studies were carried out for a 2-cell FCC using FCS-MPC strategy. The electrical parameters of the system used in the simulations are: $C=100[\mu F], R=10[\Omega]$ and $L=5[\mathrm{mH}]$. A main DC voltage of $V_{d c}=400[\mathrm{~V}]$ is considered. The FCS-MPC strategy is applied usign $h=20[\mu \mathrm{s}]$. The tuning parameters are set as $\lambda_{1}=C / 2$ and $\lambda_{2}=L / 2$.

The reference for the output current is $i_{L}^{\star}=15[A]$ and the "balanced" capacitor voltage reference is $v_{c}^{\star}=V_{d c} / 2^{1}$. This means that the desired system reference is given by $x^{\star}=$ $\left[\begin{array}{ll}200 & 15\end{array}\right]^{T}$

With these specifications one can obtain a maximum reference deviation of $\delta_{\max }=\left[\begin{array}{ll}3 & 0.59\end{array}\right]^{T}$. This implies a maximum error of $1.5 \%$ for the capacitor voltage and a maximum error of $4 \%$ for the output current. Finally, the smallest ellipse is specified by $\varepsilon=0.45 \times 10^{-3}$.

Fig. 5 depicts the start-up condition of the FCC. An initial condition of $x(0)=\left[\begin{array}{ll}0 & 0\end{array}\right]$. Normally, this system starts with the capacitor voltages previously charged in order to avoid over-voltages in the switching elements. However, it is

${ }^{1}$ Recently, we have also investigated the use of MPC to drive FCCs to out-of-balance states [13].
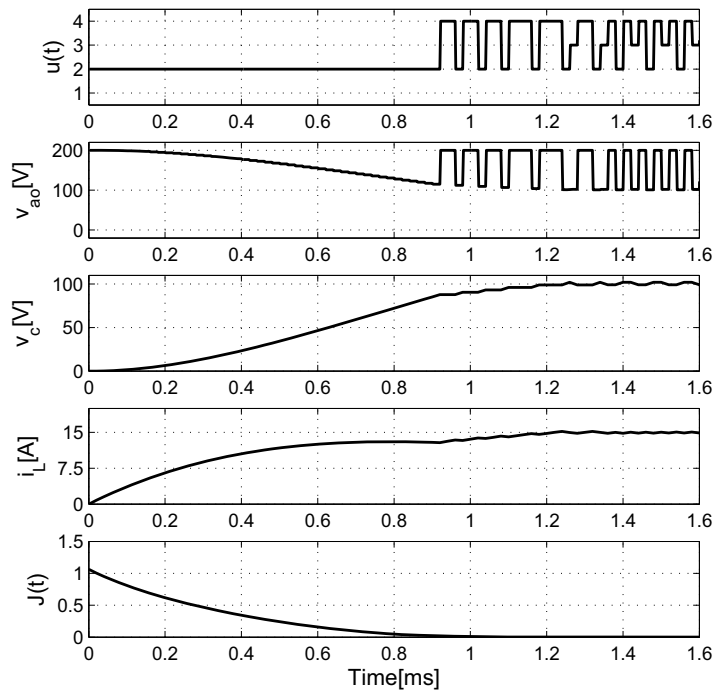

Fig. 5. Start-up condition of 2-cell FCC using FCS-MPC strategy: control input $u(t)$, output voltage $v_{a o}(t)$, capacitor voltage $v_{c}(t)$ and the output current $i_{L}(t)$ an interesting test in order to show the performance of the controller.

Here one can see the control input sequence $u(t)$ applied for the controller. Both system variables take approximately the same time to reach their references. The modulation present in the output voltage $v_{a o}(t)$ is a natural consequence of the optimal solution; no modulation stage have been used. In addition, the cost function is decreased monotonically until positive invariant set $\mathcal{D}$ is reached. This situation is clearer depicted in Fig. 6, where one can observe the reduction of the ellipses as the system state comes near to the reference.

For FCC, internal capacitor voltages $v_{c i}$ must be precisely regulated in order to protect the switching elements. For this,

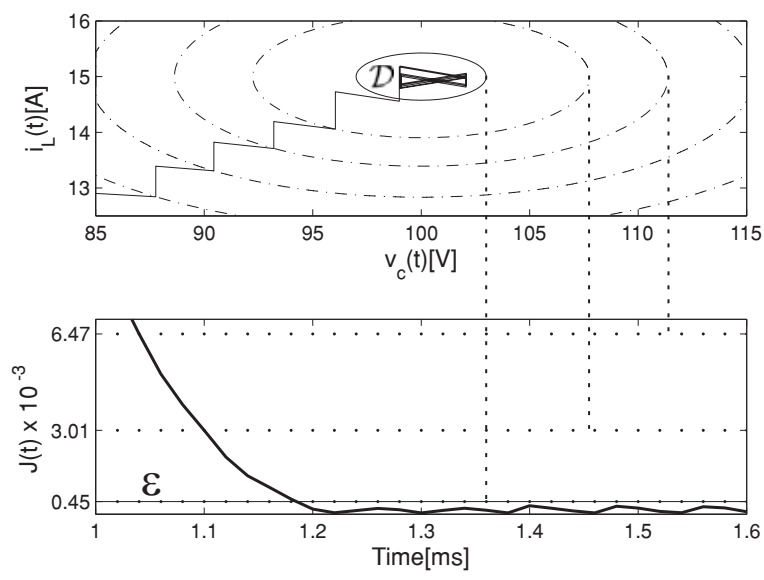

Fig. 6. Ellipsoidal trajectory of $\mathrm{J}(\mathrm{t})$ and the Positive Invariant Set $\mathcal{D}$

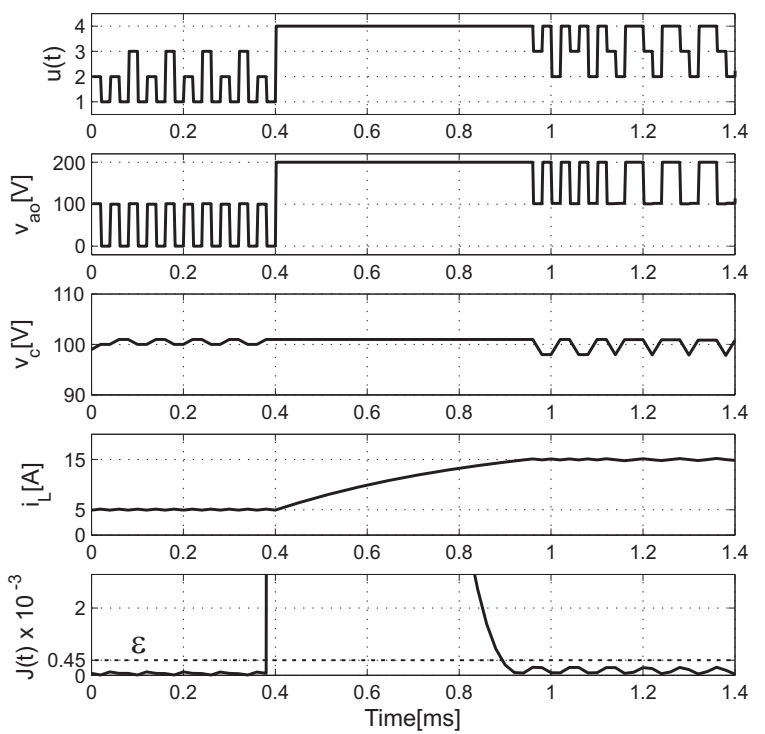

Fig. 7. Output current step: control input $u(t)$, output voltage $v_{a o}(t)$, capacitor voltage $v_{c}(t)$ and the output current $i_{L}(t)$. 
the performance of the proposed predictive strategy when a change in the output current reference $i_{L}^{\star}$ is produced is analyzed. The results of this test are presented in the Fig 7. Here the initial condition for the output current is $i_{L}=5[A]$ and $v_{c}=100[V]$. Subsequently, in the instant $t=0.4[\mathrm{~ms}]$, the current reference is set as $i_{L}=15[A]$ producing an increment in the cost function $J(t)$. As a consequence of this, the controller changes the control input pattern $u(t)$ in order to reduce the cost function until reach a value of $J(t)<\varepsilon$. When this occurs, the system remains inside of the positive invariant set $\mathcal{D}$. Despite this situation, the capacitor voltage is kept under control.

\section{CONCLUSIONS}

This paper has described preliminary work on the stability analysis of MPC based converter control system. A simple example of an FCC-MPC has been studied in detail.

It has been shown that the concepts of invariant sets are important to describe the trajectory of the system.

For this case, we have shown that always is possible to apply a control input sequence that reduces monotonically the proposed cost function. Moreover, one can find the smallest ellipsoid in which the system state will be confined. The definition of this positive invariant set allows one to determine an admissible error for the system based on the electrical parameter and the sampling period.

Simulation results for 2-cell FCC have been provided which show that FCS-MPC algorithm leads to Multicell Converters closed-loop with a favorable performance.

The presented methodology has been developed for constant reference. However, Multicell Converters are widely used in $\mathrm{AC}$ applications, consequently, it is desirable to extend this analysis for sinusoidal reference.

\section{REFERENCES}

[1] P. Cortés, M. P. Kazmierkowski, R. M. Kennel, D. E. Quevedo, and J. Rodriguez, "Predictive control in power electronics and drives," Industrial Electronics, IEEE Transactions on, vol. 55, no. 12, pp. 43124324, Dec. 2008.

[2] S. Kouro, P. Cortés, R. Vargas, U. Ammann, and J. Rodriguez, "Model predictive control: A simple and powerful method to control power converters," Industrial Electronics, IEEE Transactions on, vol. 56, no. 6, pp. 1826-1838, June 2009

[3] L. Malesani, P. Mattavelli, and S. Buso, "Robust dead-beat current control for pwm rectifiers and active filters," Industry Applications, IEEE Transactions on, vol. 35, no. 3, pp. 613-620, May/Jun 1999.

[4] G. C. Goodwin, M. M. Seron, and J. A. De Doná, Constrained Control \& Estimation: An Optimization Approach. Springer-Verlag, 2005.

[5] R. Kennel, A. Linder, and M. Linke, "Generalized predictive control (gpc)-ready for use in drive applications?" in Power Electronics Specialists Conference, 2001. PESC. 2001 IEEE 32nd Annual, vol. 4, 2001, pp. 1839-1844 vol. 4.

[6] D. Q. Mayne, J. B. Rawlings, C. V. Rao, and P. O. M. Scokaert, "Constrained model predictive control: Stability and optimality," Automatica, 2000 .

[7] G. Zhai, I. Matsune, J. Imae, and T. Kobayashi, "A note on multiple lyapunov functions and stability condition for switched and hybrid systems," in Control Applications, 2007. CCA 2007. IEEE International Conference on, Oct 2007, pp. 226-231.

[8] E. I. Silva, B. P. McGrath, D. E. Quevedo, and G. C. Goodwin, "Predictive Control of Flying Capacitor Converter," Proc. of the 2007 American Control Conference, pp. 3763-3768, Jul. 2007.
[9] F. Blanchini and S. Miani, Set-Theoretic Methods in Control. Birkhauser, 2008.

[10] T Meynard, H. Foch, P. Thomas, J. Courault, R. Jakob and M. Nahrstaedt, "Multicell converters: basic concepts and industry applications," IEEE Transactions on Industrial Electronics, vol. 49, no. 5, pp. 955-964, Oct. 2002.

[11] R. Aguilera, D. Quevedo, T. Summers, and P. Lezana, "Predictive control algorithm robustness for achieving fault tolerance in multicell converters," Nov. 2008, pp. 3302-3308.

[12] R. Aguilera and D. Quevedo, "Capacitor voltage estimation for predictive control algorithm of flying capacitor converters," in Industrial Technology, 2009. ICIT 2009. IEEE International Conference on, Feb. 2009, pp. 1-6.

[13] P. Lezana, R. Aguilera, and D. E. Quevedo, "Model predictive control of an asymmetric flying capacitor converter," Industrial Electronics, IEEE Transactions on, vol. 56, no. 6, pp. 1839-1846, June 2009. 